\title{
Expression of Invasion-Related Extracellular Matrix Molecules in Human Glioblastoma Versus Intracerebral Lung Adenocarcinoma Metastasis
}

\author{
Authors \\ I. Varga ${ }^{1}$, G. Hutóczki ${ }^{2}$, M. Petrás ${ }^{3}$, B. Scholtz ${ }^{4}$, E. Mikó ${ }^{4}$, A. Kenyeres ${ }^{5}$, J. Tóth ${ }^{6}$, G. Zahuczky ${ }^{4}$, L. Bognár ${ }^{2}$, \\ Z. Hanzély ${ }^{7}$, A. Klekner ${ }^{2}$ \\ Affiliations \\ Affiliation addresses are listed at the end of the article
}

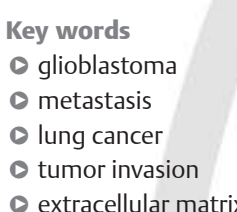

Bibliography

DOI http://dx.doi.org/

10.1055/s-0030-1249698

Published ahead of print:

15 April 2010

Cen Eur Neurosurg 2010;

71: 173-180

(c) Georg Thieme Verlag KG

Stuttgart · New York

ISSN 1868-4904

Correspondence

Dr. A. Klekner

University of Debrecen

MHSC Hungary

Department of Neurosurgery

4030 Debrecen

Nagyerdei krt 98

4032 Debrecen

Hungary

Tel.: + 36/52/419418

Fax: $+36 / 52 / 419418$

aklekner@yahoo.com

\begin{abstract}
$\nabla$

Tumor cell invasion into the surrounding brain tissue is mainly responsible for the failure of radical surgical resection, with tumor recurrence in the form of microdisseminated disease. Extracellular matrix (ECM)-related molecules and their receptors predominantly participate in the invasion process, including cell adhesion to the surrounding microenvironment and cell migration. The extent of infiltration of the healthy brain by malignant tumors strongly depends on the tumor cell type. Malignant gliomas show much more intensive peritumoral invasion than do metastatic tumors. In this study, the mRNA expression of 30 invasion-related molecules (twenty-one ECM components, two related receptors, and seven ECM-related enzymes) was investigated by quantitative reverse transcriptase-polymer-
\end{abstract}

\section{Introduction}

During malignant transformation, invasiveness is determined by complex functions of tumor cells of distinct histological types. A three-step model of invasion has been applied to a variety of malignant cell types. Tumor cells at the invasive site 1 ) detach from the growing primary tumor mass; 2 ) adhere to the extracellular matrix (ECM) via specific receptors; and 3) locally degrade the ECM components, forming a pathway for neoplastic cells to migrate into the surrounding tissue [51]. Malignant gliomas are the most common primary brain tumors. They are associated with the shortest survival time because of their infiltrative invasion of the normal brain, which makes complete removal of these tumors practically impossible. Invasive primary brain tumors are almost invariably fatal, recurring close to the resection margin in almost all cases. Primary brain tumors have a strong tendency to invade ase chain reaction. Fresh frozen human tissue samples from glioblastoma (GBM), intracerebral lung adenocarcinoma metastasis, and normal brain were evaluated. Significant differences were established for 24 of the 30 molecules. To confirm our results at the protein level, immunohistochemical analysis of seven molecules was performed (agrin, neurocan, syndecan, versican, matrix metalloproteinase 2 [MMP-2], MMP-9, and hyaluronan). Determining the differences in the levels of invasion-related molecules for tumors of different origins can help to identify the exact molecular mechanisms that facilitate peritumoral infiltration by glioblastoma cells. These results should allow the selection of target molecules for potential chemotherapeutic agents directed against highly invasive malignant gliomas.

and, with rare exceptions, do not metastasize outside the brain $[5,24,26]$. In contrast, carcinomas, which often metastasize to the brain, are more significantly and sharply delineated from the surrounding brain tissue, infiltrate only short distances, and invade as groups of cells rather than as single cells [4]. Consequently, successful total surgical resection of metastatic tumors of the brain can be achieved in many cases, whereas this is usually not possible for primary invasive brain tumors.

The ECM constitutes a considerable proportion of normal brain volume. There is much evidence that ECM components can modulate brain tumor growth, proliferation, and invasion by many different mechanisms [6]. The ECM is composed mainly of hyaluronan (HA), proteoglycans (PGs), and tenascin [12]. To allow cell adhesion and migration, the ECM components interact with specific receptors on the cell membrane, such as integrins, CD44, or CD168. Some proteases and 
synthases also strongly influence invasiveness because of their capacity to alter the actual levels of the ECM molecules or to degrade the pericellular network [4].

To understand the evident differences between glioblastoma (GBM) and intracerebral metastasis, we compared the mRNA expression of 30 invasion-related molecules in GBM and intracerebral bronchial adenocarcinoma metastases. To analyze their relationship to the environment, the same expression patterns were also determined in normal brain tissue. For morphological information and to check whether or not differences in mRNA expression appear on a post-translational level, immunohistochemical analyses were performed for seven molecules (agrin, neurocan, syndecan, versican, matrix metalloproteinase 2 [MMP-2], MMP-9, and HA).

\section{Material and Methods}

V

Tissue samples

Thirty tissue samples were collected during neurosurgical operations. The samples were frozen promptly after removal on the surface of liquid nitrogen and stored at $-80{ }^{\circ} \mathrm{C}$ until processing. Each sample was collected from a different patient. Eleven pieces of infiltrated margins of GBMs, ten pieces of intracerebral lung adenocarcinoma metastases, and nine normal brain tissue samples taken from epilepsy surgery were carefully selected by an experienced neuropathologist for further investigation. Sections for histological analysis and immunohistochemistry were cut from the same samples used for the mRNA assays. The fresh frozen tissue samples were provided by the Neurosurgical Tissue and Tumor Bank of the Neurosurgical Department of our university. All procedures were approved by the Ethical Committee and every patient signed an informed consent form.

\section{RNA analysis}

The mRNA expression of 30 ECM-related molecules was determined by real-time quantitative reverse transcriptase-polymerase chain reaction (RT-PCR): 21 PGs, three different MMPs, three hyaluronan synthases (HASs), chondroitinase $A B C$, and the two cell membrane receptors CD44 and CD168. Tumor markers (glial fibrillary acidic protein [GFAP], carcinoembryonic antigen [CEA], and cytokeratins 18 and 19) and the proliferation marker Ki-67, were also tested.

Fresh-frozen tissue samples were first pulverized with a manual CryoPress device (Microtec Co., Japan) precooled in liquid $\mathrm{N}_{2}$. The powdered still-frozen tissue was then scraped into the appropriate volume of TriReagent (Invitrogen, USA) and homogenized instantly with a rotor-stator homogenizer. Total RNA was isolated from the TriReagent lysates according to the manufacturer's instructions. RNA purity and quantity were assessed on a NanoDrop ${ }^{\circledR}$ ND-1000 spectrophotometer (NanoDrop Technologies, USA) and then stored at $-80{ }^{\circ} \mathrm{C}$. RNA quality was checked on $1.2 \%$ agarose gel stained with ethidium bromide. Total RNA was converted to single-stranded cDNA with the High-Capacity cDNA Archive Kit with RNasin (Applied Biosystems, USA) using $600 \mathrm{ng}$ of total RNA per sample in one reverse transcription reaction. The cDNA transcribed from $100 \mathrm{ng}$ of total RNA was loaded per port of the microfluidic card.

TaqMan Low Density Array (TLDA) experiments were performed using the Applied Biosystems 7900 HT real-time PCR system with the Micro Fluidic Card upgrade (Applied Biosystems, USA). The Micro Fluidic Card format allowed the analysis of 40 genes per sample, and each sample was analyzed in duplicate. The Micro Fluidic Cards were analyzed with the SDS 2.1 software as relative quantification studies (maximum 10 cards per study) with automatic threshold settings, and the $C_{T}$ values were exported for further analysis. Each 40-gene set contained three different assays for housekeeping genes: $\beta$-actin (ACTB), $\beta_{2^{-}}$ microglobulin $\left(\mathrm{B}_{2} \mathrm{M}\right)$, and glyceraldehyde 3-phosphate dehydrogenase (GAPDH). ACTB and GAPDH showed the least variation among the samples and were used as the reference genes to calculate the $\mathrm{dCt}$ value for each gene.

Expression values were calculated using the comparative $C_{T}$ method, as described previously [23]. Briefly, assuming that the PCR efficiency for any gene on the TLDA is close to 1, mRNA expression [15] for a given gene in the tumor or normal (calibrator) sample can be compared using the simplified equations [17]:

$\mathrm{X}_{\text {tumor }}=2^{-\mathrm{dCTtumor}}$ and $\mathrm{X}_{\text {normal }}=2^{-\mathrm{dCTnormal}}$

$2^{-\mathrm{dCT}}$ values are the input expression values during further analysis with GeneSpring 7.3 software (Silicon Genetics, Redwood City, CA, USA). Instead of calculating the expression values ( $2^{\text {-ddCT }}$ ) relative to those of the normal samples, this allowed inspection of individual mRNA expression (X) differences within sample categories. To identify genes with significantly different expression levels between different sample groups Mann-Whitney $U$-test was performed. Significance was established at $p<0.05$.

\section{Immunohistochemistry}

After evaluating the results of the mRNA determination seven molecules were selected and investigated at the protein level with immunohistochemistry: agrin, neurocan, syndecan, versican, MMP-2, -9, and hyaluronan.

Frozen and stored $\left(-80^{\circ} \mathrm{C}\right)$ samples were fixed in Saint Marie's fixative $[37,44]$ for $24 \mathrm{~h}$ at $4{ }^{\circ} \mathrm{C}$. After fixation and dehydration, the tissue samples were embedded in wax, and $5 \mu \mathrm{m}$ sections were cut. The sections were stained with hematoxylin-eosin and immunohistochemical reactions were carried out according to the following protocol. Slides were preincubated in ready-to-use (2.5\%) normal horse serum (Vector, Burlingame, CA, USA) for $30 \mathrm{~min}$ at $37^{\circ} \mathrm{C}$ to prevent nonspecific binding of the primary antibodies. The sections were then incubated overnight at $4{ }^{\circ} \mathrm{C}$ with the appropriately diluted antibodies. To promote the morphological evaluation a supplementary section for immunohistochemical and routine hemalaun staining was also performed. The immunohistochemical and HA reactions were visualized with the avidin-biotin-peroxidase complex, and the peroxidase was detected with a solution containing the $\mathrm{H}_{2} \mathrm{O}_{2}$ substrate and the diaminobenzidine (DAB) chromogen (ImmPress Reagent Kit, Vector; Peroxidase Substrate DAB Kit, Vector). Finally, the nuclei were labeled with hemalaun staining, and the sections were mounted in DePeX (BDH Laboratory Supplies, Poole, UK). To control for the specificity of the immunohistochemical reactions, sections were treated with the same protocol, excluding the primary antibodies, which were replaced with nonimmune IgG (Sigma, St Louis, MO, USA) solution at the optimal dilution for the different primary antibodies. HA was detected with a biotinylated HA-binding complex probe (bHABC), which contains the hyaluronan-binding G1 domain of the cartilage PG, aggrecan, and link protein, which have high affinity and specificity for HA [42]. The bHABC probe was kindly provided by 
R. Tammi and M. Tammi (Institute for Biomedicine, Department of Anatomy, University of Kuopio, Kuopio, Finland). The probe was used at a concentration of $5 \mu \mathrm{g} / \mathrm{mL}$, and the reaction was performed as described previously [9].

The reactions were evaluated by three different investigators experienced in histology on a score of 1-5 in three different locations: intracellular, cell-membrane, and extracellular regions, which were evaluated separately for morphological information. The scores of the regions were then summed for general comparison.

\section{Results}

Thirty tissue samples were investigated from three different homogeneous groups of tissues: eleven GBM samples originating from the infiltrated margins of the tumor, ten intracerebral lung adenocarcinoma metastases, and nine normal brain tissues. The results of the statistical analysis for differences in mRNA expression levels of 34 molecules between the investigated sample groups are summarized in $\bullet$ Table 1.

mRNA expression level

The mRNA expression of the tumor markers and the Ki67 proliferation marker confirmed the histological diagnosis. The
mRNA expression of the investigated molecules is illustrated in $\odot$ Fig. 1.

\section{GBM versus normal brain}

The expression of mRNAs for agrin, fibronectin, laminin $\alpha-4, \beta-1$ and $\beta-2$, perlecan, syndecan- 1 , tenascin-C, CD44, CD168, HAS-2, MMP-2 and -9 was significantly elevated in GBM relative to that in the normal brain, whereas the mRNA expression of syndecan4 , tenascin-R and HAS-1 was statistically lower in the GBM. The mRNA levels of aggrecan, brevican, laminin $\alpha-1,-2$, laminin $\beta-1$, laminin $\gamma-1$, matrilin- 1 and -2 , neurocan, neuroglycan, syndecan-2, versican, chondroitinases, HAS-3, and MMP-8 did not differ significantly between normal brain and GBM.

\section{Intracerebral lung adenocarcinoma metastases versus}

normal brain

Significantly reduced mRNA expression of brevican, laminin $\alpha-1$, matrilin-2, neurocan, neuroglycan-C, syndecan-2, tenascin- $R$, and HAS-1 was detected in lung adenocarcinoma metastases compared with that in normal brain. Conversely, the expression of agrin, fibronectin, laminin $\beta-1, \beta-2$ and $\gamma-1$, perlecan, syndecan-1 and -4, CD168 and MMP-9 mRNAs was statistically elevated in the metastatic tumor relative to that in normal brain. There was no apparent difference in the expression of aggrecan, laminin $\alpha-2,-4$, matrilin- 1 , tenascin-C, versican, CD44, chon-

Table 1 Significant differences (bold) in the mRNA expression levels of 30 invasion-related molecules and five tumor markers in glioblastoma (GBM), intracerebral lung adenocarcinoma metastasis (Met), and normal brain tissues (Norm).

\begin{tabular}{|c|c|c|c|c|c|c|}
\hline & \multicolumn{2}{|c|}{ Norm - Met } & \multicolumn{2}{|c|}{ Norm - GBM } & \multicolumn{2}{|c|}{ GBM - Met } \\
\hline & p-value & fold change & p-value & fold change & p-value & fold change \\
\hline aggrecan & 0.421 & 0.79 & 0.421 & 0.39 & 1.000 & 2.01 \\
\hline agrin & 0.002 & 0.30 & 0.012 & 0.44 & 0.218 & 0.67 \\
\hline brevican & $<0.001$ & 172.46 & 0.820 & 1.27 & $<0.001$ & 135.66 \\
\hline fibronectin & $<0.001$ & 0.08 & $<0.001$ & 0.09 & 0.916 & 0.87 \\
\hline laminin alfa-1 & 0.032 & 4.55 & 0.548 & 2.98 & 0.421 & 1.53 \\
\hline laminin alfa-2 & 0.310 & 1.84 & 0.841 & 0.82 & 0.222 & 2.24 \\
\hline laminin alfa-4 & 0.713 & 0.85 & 0.023 & 0.47 & 0.098 & 1.79 \\
\hline laminin beta-1 & 0.020 & 0.36 & 0.001 & 0.27 & 0.379 & 1.33 \\
\hline laminin beta-2 & 0.020 & 0.44 & 0.005 & 0.34 & 0.130 & 1.29 \\
\hline laminin gamma-1 & 0.016 & 0.29 & 0.222 & 0.38 & 1.000 & 0.76 \\
\hline matrilin-1 & 0.548 & 1.69 & 0.310 & 2.34 & 1.000 & 0.72 \\
\hline matrilin-2 & 0.002 & 6.07 & 0.761 & 0.90 & $<0.001$ & 6.72 \\
\hline neurocan & $<0.001$ & 44.76 & 0.095 & 2.29 & 0.001 & 19.50 \\
\hline neuroglycan-C & $<0.001$ & 46.94 & 0.058 & 3.15 & $<0.001$ & 14.89 \\
\hline perlecan & $<0.001$ & 0.08 & $<0.001$ & 0.12 & 0.342 & 0.70 \\
\hline syndecan-1 & $<0.001$ & 0.01 & 0.008 & 0.23 & $<0.001$ & 0.06 \\
\hline syndecan-2 & 0.008 & 3.27 & 1.000 & 1.44 & 0.151 & 2.27 \\
\hline syndecan-4 & 0.020 & 0.41 & 0.048 & 1.53 & 0.008 & 0.27 \\
\hline tenascin- $C$ & 0.348 & 0.40 & 0.006 & 0.06 & 0.007 & 6.91 \\
\hline tenascin- $R$ & $<0.001$ & 136.10 & $<0.001$ & 10.53 & 0.018 & 12.92 \\
\hline versican & 0.596 & 1.27 & 0.289 & 0.56 & 0.307 & 2.29 \\
\hline CD44 & 0.094 & 0.39 & 0.003 & 0.15 & 0.012 & 2.70 \\
\hline CD168 & $<0.001$ & 0.06 & $<0.001$ & 0.12 & 0.053 & 0.46 \\
\hline chondroitinases (AC.ABC) & 0.222 & 0.77 & 0.841 & 0.95 & 0.690 & 0.81 \\
\hline hyaluronan synthase-1 & $<0.001$ & 26.98 & $<0.001$ & 33.55 & 0.504 & 0.80 \\
\hline hyaluronan synthase- 2 & 0.838 & 0.77 & 0.002 & 0.11 & 0.003 & 6.84 \\
\hline hyaluronan synthase-3 & 0.421 & 1.75 & 0.690 & 0.83 & 0.222 & 2.10 \\
\hline MMP-2 & 0.131 & 0.37 & 0.004 & 0.15 & 0.032 & 3.08 \\
\hline MMP-8 & 1.000 & 0.89 & 0.857 & 1.50 & 0.310 & 0.60 \\
\hline MMP-9 & 0.001 & 0.06 & $<0.001$ & 0.05 & 0.805 & 1.11 \\
\hline cytokeratin 18 & 0.008 & 0.002 & 0.310 & 0.72 & 0.008 & 0.003 \\
\hline cytokeratin 19 & 0.008 & 0.003 & 0.151 & 4.49 & 0.008 & 0.001 \\
\hline GFAP & 0.016 & 33.31 & 0.548 & 0.73 & 0.008 & 45.61 \\
\hline Ki67 & 0.008 & 0.01 & 0.008 & 0.01 & 0.548 & 0.82 \\
\hline
\end{tabular}




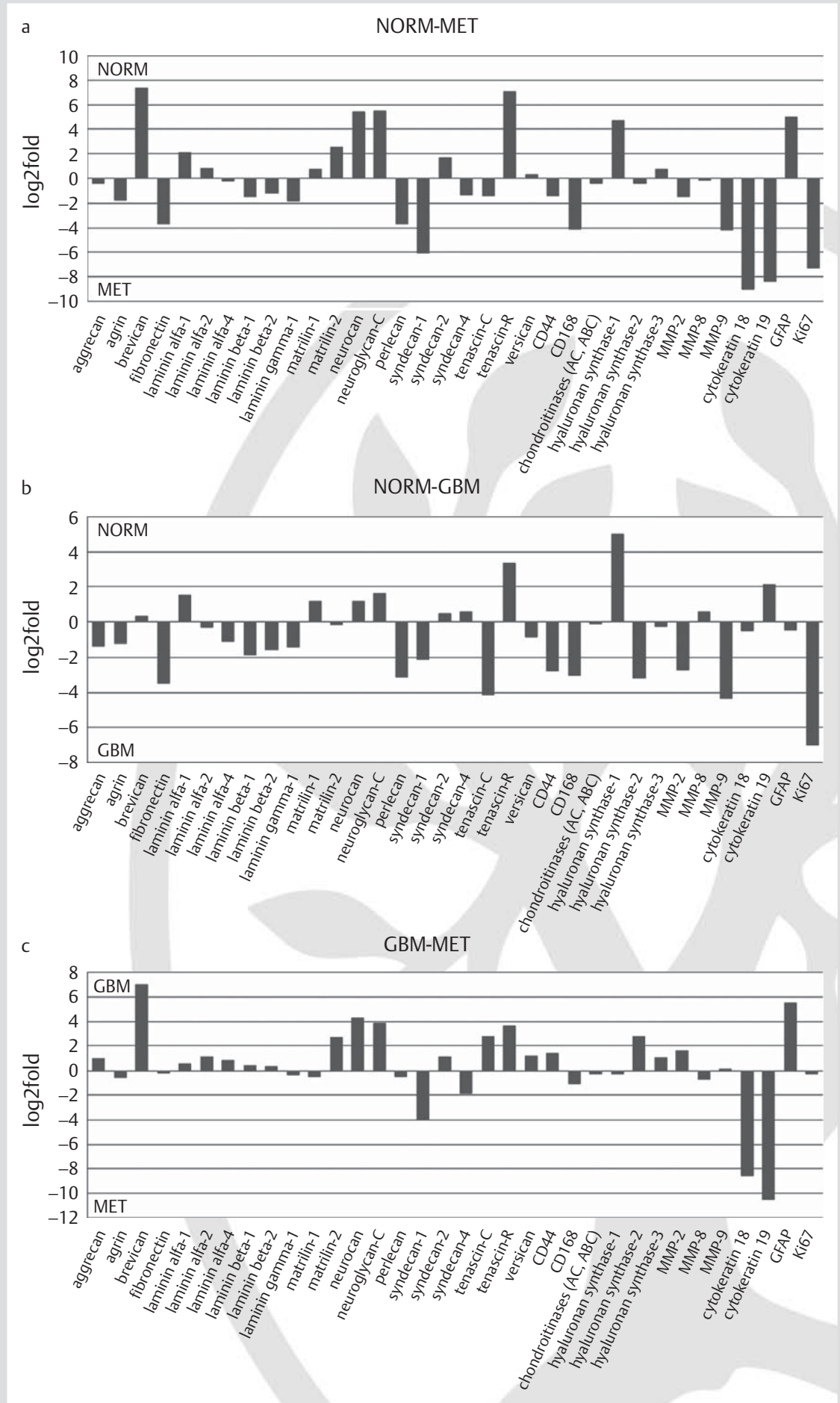

Fig. 1 mRNA expression of 30 invasion-related molecules, tumor markers and Ki67 proliferation marker in glioblastoma (GBM), intracerebral lung adenocarcinoma metastasis (Met), and normal brain (Norm). Relation between mRNA expression of each molecule (fold change) in normal brain and in metastasis (a); normal brain and GBM (b); GBM and metastasis (c).

droitinases, HAS-2, -3 , MMP-2 and -8 transcripts between the two types of tissue.

\section{GBM versus intracerebral lung adenocarcinoma} metastases

In a comparison of the anaplastic tumors of different origins, the mRNA expression of brevican, matrilin-2, neurocan, neuroglycan-C, tenascin-C and R, CD44, HAS-2 and MMP-2 was significantly higher in GBM compared to lung adenocarcinoma metastases. The transcripts of syndecan- 1 and syndecan- 4 were significantly elevated in the intracerebral adenocarcinoma. We detected no statistically significant differences in agrin, aggrecan, fibronectin, laminin $\alpha-1,-2$ or -4 , laminin $\beta-1,-2$, laminin $\gamma-1$, matrilin-1, perlecan, syndecan-2, versican, CD168, chondroitinase, HAS- 1 and -3 , or MMP-8, and -9 transcripts.

\section{Immunohistochemistry}

The immunohistochemical and HA histochemical reactions are summarized in 0 Fig. 2. According to the morphological evaluation, immunoreactivity for agrin, neurocan, syndecan, and versican was most intense mainly on the cell membrane and in the extracellular space, whereas the MMPs showed strong immunoreactivity on the cell membrane and intracellularly. Most intense immunostaining of agrin, syndecan and MMP-9 was observed in the lung adenocarcinoma metastases, while MMP-2, neurocan and hyaluronan showed the highest values in GBM samples. The 


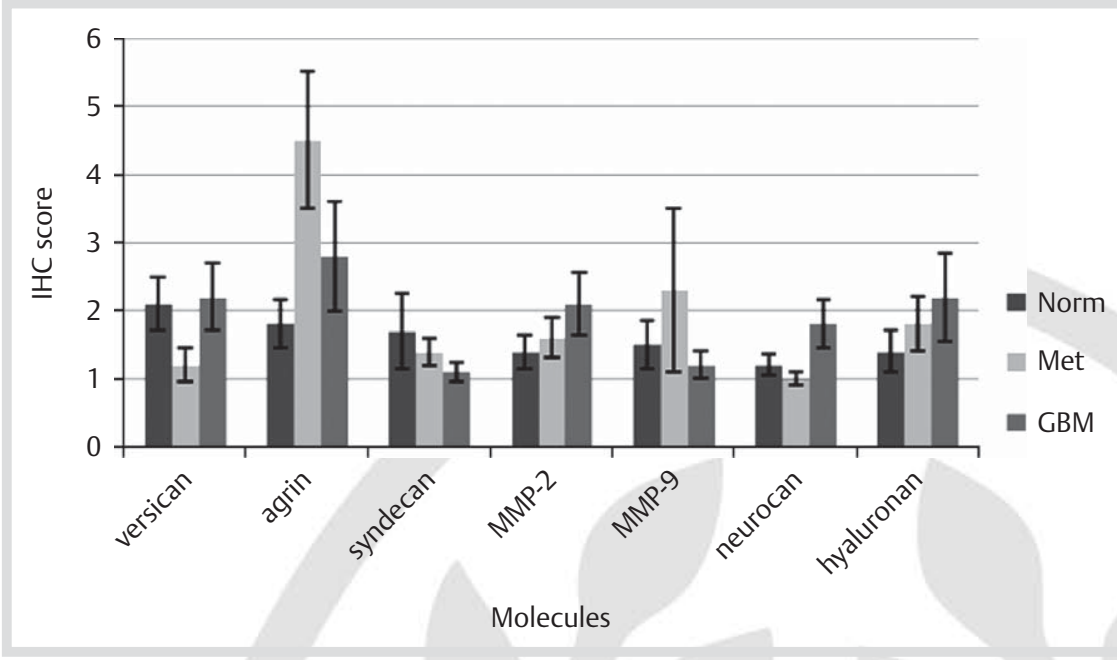

Fig. 2 Immunohistochemical (IHC) evaluation of four proteoglycans, two matrix metalloproteinases, and hyaluronic acid in glioblastoma, intracerebral lung adenocarcinoma metastasis (Met), and normal brain (Norm). The reactions were evaluated by three different investigators experienced in histology on a score of $1-5$ in three different regions. The scores of the regions were then summed for general comparison. staining intensity of tumor markers and Ki-67 was in accordance with the histology.

\section{Discussion \\ $\nabla$}

Gliomas are the most common primary intracranial tumors. The incidence of brain metastasis is about double the number of primary brain tumors, and more than half of them originate from lung cancer [11]. Both tumor types are anaplastic, dedifferentiated neoplasms, but their biological behaviour and thus their therapeutic management differs considerably. The unambiguous ability of high grade gliomas to invade normal neural tissue makes them difficult to control and makes complete surgical resection almost impossible. This also explains the extremely high mortality associated with anaplastic gliomas. In contrast, the invasiveness of intracerebral metastases of anaplastic tumors is much more moderate, and radical surgical removal can be achieved in most cases.

The extracellular matrix plays a pivotal role in the tumorous infiltration of the surrounding tissue. The presence and functions of hyaluronic acid, PGs, synthetic and lytic enzymes, and specific adhesion receptors have already been intensively investigated to clarify the molecular mechanisms of invasion, and a positive correlation has been established many times $[4,17,18,22,31,39]$.

To identify the molecules that are probably responsible for the distinct invasiveness of high-grade gliomas and metastatic tumors, the mRNA expression of 30 invasion-related molecules was measured in fresh-frozen neurosurgical tissue samples of GBM, intracerebral lung adenocarcinoma metastases, and normal brain tissue. Based on data from the literature, 21 ECM components, seven proteases, the membrane receptor for hyaluronan (CD44), and CD168 were selected for quantitative RT-PCR. To verify the results of mRNA analysis, immunohistochemical analysis of seven molecules (agrin, neurocan, syndecan, versican, MMP-2, MMP-9, and hyaluronan) was performed. The mRNA expression patterns of the analyzed chosen molecules are illustrated in $\odot$ Fig. 1.

Previous studies have described a positive correlation between the invasion potential of gliomas and the expression of brevican $[10,46]$, fibronectin $[25,43]$, laminin $[13,25]$, syndecan [48], tenascin-C [16,52], versican [32], MMP-9 [3,40,53], hyaluronan [7], and CD44 $[30,35,49]$. We also detected significant differ- ences in the mRNA profiles of normal brain and GBM for fibronectin, laminin $\beta-1$, perlecan, syndecan- 1 , -4 , tenascin-C, $\mathrm{R}, \mathrm{CD} 44, \mathrm{CD} 168$, HAS-1, -2 , and MMP-2 and -9 . There are no relevant data in the literature relating astrocytomas to changes in the levels of aggrecan, matrilin, perlecan, neuroglycan- $C$, neurocan, CD168, or chondroitinase. In this study the mRNA expression of perlecan and CD168 was significantly higher in gliobastomas compared to normal brain, while there were no evident differences between the others.

ECM components and invasion-related molecules have also been studied in some cases of non-small-cell lung carcinoma. In bronchial adenocarcinomas, elevated levels of hyaluronan [33], fibronectin [15,19], laminin [28,41], versican [34], MMP-9 [3], MMP-2 [8], and CD44 [21] have been reported, whereas increased levels of perlecan, tenascin- $C$, and syndecan do not correlate well with tumor progression $[14,27,38]$. There are no data supporting a definite correlation between agrin, brevican, matrilin, neuroglycan-C, neurocan, CD168, chondroitinase, or HAS and the tumor behavior of lung adenocarcinoma. Comparing the intracerebral lung adenocarcinoma metastases with normal brain, we detected a significant difference in the mRNA expression of 18 molecules out of the 30 tested ( $\bullet$ Table 1 ). There are some molecules that are specific for the tissue of origin (e.g. brevican, neurocan, neuroglycan-C in the brain samples), while others probably play an important role in peritumoral invasion (fibronectin, syndecan-1, -4, CD168 and MMP-9). But the explanation for the high expression of agrin, laminin $\beta-1, \beta-2, \gamma-1$ and perlecan in intracerebral lung adenocarcinoma metastasis needs further investigation.

The mRNA expression of 11 molecules differed significantly between GBM and lung adenocarcinoma metastases ( $\bullet$ Table 1 ). Since brevican, matrilin-2, neurocan, neuroglycan- $\mathrm{C}$ and tenascin-R had a higher mRNA expression both in normal brain and in GBM compared to the metastatic tumors, these molecules can be interpreted as specific molecules for glial tissues. On the other hand, the mRNA expression of tenascin-C, CD44, HAS-1 and MMP-2 was elevated only in the GBM compared to the metastatic tissue. Based on these observations the high level of the former molecules might help to understand the role of ECM in GBM in avoiding antitumoral reactions of the surrounding brain tissue. Furthermore, the role of the latter molecules in the difference in invasion effectiveness of GBM can be also hypothesized. 
Lung adenocarcinoma metastases had high expression of syndecan- 1 and -4 , but this proved to be insufficient for invading the peritumoral brain.

BEHAB (brain enriched hyaluronan binding)/brevican, a brainspecific chondroitin sulfate PG, shows dramatic upregulation in gliomas and it is also upregulated during periods of increased glial cell motility during development and following brain injury [29]. Viapiano et al. established that brevican expression in gliomas have a restricted membrane localization, and its presence in high-grade gliomas suggests that it may play a significant role in glioma progression, making it an important new potential therapeutic target [45]. We detected no significant difference in mRNA expression of brevican between the normal brain and GBM, thus our data does not support the role of brevican in GBM progression.

Although the mRNA expression of fibronectin was found to be significantly higher in GBM than in normal brain, it was also definitely high in lung adenoracinoma metastases as well, so that the role of fibronectin in the different invasion activity of the two different tumor types is not supported by our results.

Neurocan, a chondroitin-sulphate PG, is involved in the promotion of neurite outgrowth through interaction with heparan-sulphate PGs [1]. We found significantly lower mRNA expression of neurocan in the lung adenocarcinoma metastases than in GBM, but the highest value was detected in the normal brain, so the role of neurocan in the invasion process of GBM is not clear yet. Based on our results it could be hypothesized that neurocan plays a predominant part in cell differentiation and not in cell proliferation or migration. The reason might be that neurocan promotes normal neurite outgrowth and not tumoral membrane processes. Further investigations are needed for a more precise explanation.

Neuroglycan-C, another chondroitin-sulphate PG, is a ligand of epidermal growth factor receptor (EGFR) Erb-B3 and thus plays an active role in tumor growth $[2,20]$. The mRNA expression of neuroglycan- $C$ was found to be significantly lower in lung adenocarcinoma metastases than in the other two groups, but the highest value was detected in normal brain, which does not support its putative role in glioma invasion.

Syndecans were previously found to play a part in glioma invasion [48]. We found the highest mRNA expression of snydecan-1 and 4 in lung adenocarcinoma metastases and syndecan-2 in normal brain. Based on these data we can not determine their specific role in the extremely high infiltrative activity of GBM. Tenascin- $\mathrm{C}$ is a glycoprotein of the ECM that was reported to be positively correlated to glioma invasion $[9,27]$ and have a negative correlation with tumor progression of bronchial adenocarcinomas $[14,27,38]$. Since we also detected a higher mRNS expression of tenascin-C in GBM than in lung adenocarcinoma metastases, and it was low again in normal brain, a specific role of tenascin-C in glioma invasion can be strongly hypothesized.

Versican is one of the most common ECM components in the brain and plays a role in neurite outgrowth, cell attachment and EGFR-mediated signal pathways [50]. Its elevated expression in gliomas has been described previously [32]. We detected elevated mRNA expression of versican in GBM compared to lung adenocarcinoma metastases and normal brain, but the difference was not confirmed stastically.

The positive correlation between CD44 and glioma grading and the functional connection between HA and CD44 has been reported many times $[30,35,49]$, and a similar association is also known with regard to lung adenocarcinomas [22]. In our study we detected significantly elevated CD44mRNS expression in GBM compared to lung adenocarcinoma metastases with a low CD44 mRNS expression in normal brain, indicating that CD44 may play an important role in the invasion process of malignant gliomas. HAS-2 also showed increased mRNA expression in GBM compared to lung adenocarcinoma metastases or normal brain, and immunostaining of HA was most intensive in GBM. These observations emphasize the common function of CD44 and HAS-2 in tumor infiltration of the surrounding brain.

The role of metalloproteinases in tumor invasion and their representative subtypes in gliomas and bronchial adenocarcinomas has already been described $[3,8,40,53]$. Our observation that mRNA expression of MMP-2 is significantly higher in GBM than in lung adenocarcinoma metastases and normal brain definitely underlines its role in the different invasiveness of the two different tumor types. The immunostaining profile of MMP-2 was in accordance with the results of the investigations into mRNA expression.

\section{Comparison of mRNA expression and immunohistochemical findings}

To investigate the appearance of the different mRNA expressions on a post-translational level and obtain morphological information, immunohistochemical analyses were performed for seven molecules (agrin, neurocan, syndecan, versican, matrix metalloproteinase 2 [MMP-2], MMP-9, and HA).

The changes in the intensity of immunostaining between the three tissue sample groups correlated well with the changes in mRNA expression for versican, agrin, syndecan and MMP-2 (๑ Fig. 2). In immunohistochemical analysis, agrin, syndecan and MMP-9 were predominant in bronchial adenocarcinoma, whereas MMP-2, neurocan and hyaluronan showed the greatest intensity of immunostaining in GBM.

Interestingly, the highest staining intensity and mRNA expression of agrin was detected in the metastasis group. Agrin is an important component of the blood-brain barrier [36,47], but its presence and role in intracerebral lung adenocarcinoma metastases has not yet been clarified.

MMP-9 showed the strongest immunostaining intensity in lung adenocarcinoma metastases, but its highest mRNA expression was measured in GBM. The highest mRNA expression of neurocan was detected in normal brain, but immunohistochemistry showed a slightly increased staining intensity in the tumors. These findings are probably attributable to post-transcriptional events, but further investigation is required.

HA was found to be mostly present in GBM. Since the mRNA expression of its receptor CD44 was also highest in GBM, their common role in the invasion process can be affirmed.

\section{Conclusions}

$\nabla$

By comparing the mRNA expression of 30 invasion-related molecules in GBM, normal brain, and intracerebral lung adenocarcinoma metastasis, some molecules that probably play a role in the extremely high invasive activity of GBM could be identified. Based on these results, tenascin-C, CD44, and MMP-2 seem to be predominantly involved in peritumoral infiltration by GBM, but a clear role of fibronectin and syndecans in the different infiltrative activity of these tumors could not be confirmed. Adding 
new data to previous observations, the levels of brevican, neurocan, neuroglycan-C and matrilin-2 were also shown to differ significantly between lung adenocarcinoma metastasis and GBM. Because ECM components, proteases, and their receptors have been shown to play an important role in tumor cell migration and invasion, each identified component could be investigated further as a potential target for anticancer therapy.

\section{Acknowledgements}

This study was supported by the Hungarian Ministry of Education (OTKA, no. F-049050).

\section{Conflict of Interest: None}

\section{Affiliations}

${ }^{1}$ Kenezy Hospital, Debrecen, Department of Pulmonology, Debrecen, Hungary

${ }^{2}$ University of Debrecen, Department of Neurosurgery, Debrecen, Hungary ${ }^{3}$ Medical and Health Science Center, University of Debrecen, Biophysics and Cell Biology, Debrecen, Hungary

${ }^{4}$ University of Debrecen, Department of Biochemistry and Molecular Biology, Debrecen, Hungary

${ }^{5}$ University of Debrecen, Department of Anatomy, Histology and Embryology, Debrecen, Hungary

${ }^{6}$ University of Debrecen, Department of Oncology, Debrecen, Hungary ${ }^{7}$ National Institute of Neurosurgery, Department of Pathology, Budapest, Hungary

\section{References}

1 Akita K, Toda M, Hosoki Y et al. Heparan sulphate PGs interact with neurocan and promote neurite outgrowth from cerebellar granule cells. Biochem J 2004; 383: 129-138

2 Aono S. Expression and identification of a new splice variant of neuroglycan $C$, a transmembrane chondroitin sulfate $P G$, in the human brain. J Neurosci Res 2006; 83: 110-118

3 Arnold SM. Expression of p53, bcl-2, E-cadherin, matrix metalloproteinase- 9 , and tissue inhibitor of metalloproteinases- 1 in paired primary tumors and brain metastasis. Clin Cancer Res 1999; 5: 4028-4033

4 Bellail AC, Hunter SB, Brat DJ et al. Microregional extracellular matrix heterogeneity in brain modulates glioma cell invasion. Int J Biochem Cell Biol 2004; 36: 1046-1069

5 Burger PC, Scheithauer BW. Tumors of the central nervous system. Atlas of Tumor Pathology, third series, fascicle 1994; 10: 349-369

6 Czirok A, Zamir EA, Filla MB et al. Extracellular matrix macroassembly dynamics in early vertebrate embryos. Curr Top Dev Biol 2006; 73: 237-258

7 Delpech B, Maingonnat C, Girard N et al. Hyaluronan and hyaluronectin in the extracellular matrix of human brain tumor stroma. Eur J Cancer 1993; 29: 1012-1017

8 Fears CY, Gladson CL, Woods A. Syndecan-2 is expressed in the microvasculature of gliomas and regulates angiogenic processes in microvascular endothelial cells. J Biol Chem 2006; 281: 14533-14536

9 Felszeghy S, Hyttinen M, Tammi R et al. Quantitative image analysis of hyaluronan expression in human tooth germs. Eur J Oral Sci 2000; 108: 320-326

10 Gary SC, Hockfield S. BEHAB/brevican: an extracellular matrix component associated with invasive glioma. Clin Neurosurg 2000; 47: 72-82

11 Gavrilovic IT, Posner JB. Brain metastases: epidemiology and pathophysiology. J Neuro-Oncol 2005; 75: 5-14

12 Gladson CL. The extracellular matrix of gliomas: Modulation of cell function. J Neuropath Exp Neurol 1999; 58: 1029-1040

13 Guo P, Imanishi Y, Cackowski FC et al. Up-regulation of angiopoietin-2, matrix metalloprotease-2, membrane type 1 metalloprotease, and laminin 5 gamma 2 correlates with the invasiveness of human glioma. Am J Pathol 2005; 166: 877-890

14 Han JY, Kim HS, Lee SH et al. Immunohistochemical expression of integrins and extracellular matrix proteins in non-small cell lung can- cer: correlation with lymph node metastasis. Lung Cancer 2003; 41: 65-70

15 Han S, Sidell N, Roman J. Fibronectin stimulates human lung carcinoma cell proliferation by suppressing p21 gene expression via signals involving Erk and Rho kinase. Cancer Lett 2003; 219: 71-81

16 Higuchi M, Ohnishi T, Arita $N$ et al. Expression of tenascin in human gliomas: Its relation to histological malignancy. Acta Neuropath (Berlin) 1993; 85: 481-487

17 Hirose J, Kawashima H, Yoshie O et al. Versican interacts with chemokines and modulates cellular responses. J Biol Chem 2001; 276: 5228-5234

18 Jung S, Moon KS, Kim ST et al. Increased expression of intracystic matrix metalloproteinases in brain tumors: relationship to the pathogenesis of brain tumor-associated cysts and peritumoral edema. J Clin Neurosci 2007; 14: 1192-1198

19 Khan ZA, Caurtero J, Barbin YP Chan BM et al. ED-B fibronectin in nonsmall cell lung carcinoma. Exp Lung Res 2005; 31: 701-711

20 Kinugasa Y. Neuroglycan C, a novel member of the neuregulin family. Biochem Biophys Res Commun 2004; 321: 1045-1049

21 Lee LN, Kuo SH, Lee YC et al. CD44 splicing pattern is associated with disease progression in pulmonary adenocarcinoma. J Formos Med Assoc 2005; 104: 541-548

22 Leivonen $M$, Lundin J, Nordling S et al. Prognostic value of syndecan-1 expression in breast cancer. Oncology 2004; 67: 11-18

23 Livak KJ, Schmittgen TD. Analysis of relative gene expression data using real-time quantitative PCR and the 2 (-Delta Delta C (T)) method. Methods 2001; 25: 402-408

24 Louis DN, Ohgaki H, Wiestler OD et al. The 2007 WHO classification of tumours of the central nervous system. Acta Neuropathol 2007; 114: 197-109

25 Mahesparan R, Read TA, Lund-Johansen M et al. Expression of extracellular matrix components in a highly infiltrative in vivo glioma model. Acta Neuropathol 2003; 105: 49-57

26 Miliaras G, Tsitsopoulos PP, Markoula $S$ et al. Multifocal glioblastoma with remote cutaneous metastasis: a case report and review of the literature. Cen Eur Neurosurg 2009; 70: 39-42

27 Nackaerts K, Verbeken E, Deneffe G et al. Heparan sulfate PG expression in human lung-cancer cells. Int J Cancer 1997; 74: 335-345

28 Niki T, Kohno T, Iba S et al. Frequent co-localization of Cox-2 and laminin-5 gamma2 chain at the invasive front of early-stage lung adenocarcinomas. Am J Pathol 2002; 160: 1129-1141

29 Nutt CL, Matthews RT, Hockfield S. Glial tumor invasion: a role for the upregulation and cleavage of BEHAB/brevican. Neuroscientist 2001; 7: 113-122

$30 \mathrm{Oz} \mathrm{B}$, Karayel FA, Gazio NL et al. The distribution of extracellular matrix proteins and CD44S expression in human astrocytomas. Path Oncol Res 2000; 6: 118-124

31 Pakula $R$, Melchior A, Denys A et al. Syndecan-1/CD147 association is essential for cyclophilin B-induced activation of p44/42 mitogen-activated protein kinases and promotion of cell adhesion and chemotaxis. Glycobiology 2007; 17: 492-503

32 Paulus $W$. Differential expression of versican isoforms in brain tumors. J Neuropathol Exp Neurol 1996; 55: 528-533

33 Pirinen $R$, Leinonen $T$, Bohm J et al. Versican in nonsmall cell lung cancer: relation to hyaluronan, clinicopathologic factors, and prognosis. Hum Pathol 2005; 36: 44-50

34 Pirinen $R$, Tammi $R$, Tammi $M$ et al. Prognostic value of hyaluronan expression in non-small-cell lung cancer: Increased stromal expression indicates unfavorable outcome in patients with adenocarcinoma. Int J Cancer 2001; 95: 12-17

35 Ranuncolo SM, Ladeda V, Specterman $S$ et al. CD44 expression in human gliomas. J Surg Oncol 2002; 79: 30-35

36 Rascher G, Fischmann A, Kroger $S$ et al. Extracellular matrix and the blood-brain barrier in glioblastoma multiforme: spatial segregation of tenascin and agrin. Acta Neuropathol (Berl) 2002; 104: 85-91

37 Sainte-Marie G. A paraffin embedding technique for studies employing immunofluorescence. J Histochem Cytochem 1962; 10: 250-256

38 Shah L, Walter KL, Borczuk AC et al. Expression of syndecan-1 and expression of epidermal growth factor receptor are associated with survival in patients with nonsmall cell lung carcinoma. Cancer 2004; 101: $1632-1638$

39 Shibata Sh, Fukada K, Suzuki Sh et al. Histochemical localisation of versican, aggrecan and hyaluronan in the developing condylar cartilage of the fetal rat mandible. J Anat 2001; 198: 129-135

40 Sunami E, Tsuno N, Osada T et al. MMP-1 is a prognostic marker for hematogenous metastasis of colorectal cancer. The Oncologist 2000 5: $108-114$ 
41 Szelachowska J, Jelen M, Kornafel J. Prognostic significance of intracellular laminin and Her2/neu overexpression in non-small cell lung cancer. Anticancer Res 2006; 26: 3871-3876

42 Tammi R, Ripellino JA, Margolis RU et al. Hyaluronate accumulation in human epidermis treated with retinoic acid in skin organ culture. J Invest Dermatol 1989; 92: 326-332

43 Tews DS. Adhesive and invasive features in gliomas. Pathol Res Pract 2000; 196: 701-711

44 Tuckett F, Morriss-Kay G. Alcian blue staining of glycosaminoglycans in embryonic material: effect of different fixatives. Histochem J 1988; 20: $174-182$

45 Viapiano MS, Bi WL, Piepmeier J et al. Novel tumor-specific isoforms of BEHAB/brevican identified in human malignant gliomas. Cancer Res 2005; 65: 6726-6733

46 Viapiano MS, Hockfield S, Matthews RT. BEHAB/brevican requires ADAMTS-mediated proteolytic cleavage to promote glioma invasion. J Neurooncol 2008; 88: 261-272

47 Warth A. Redistribution of the water channel protein aquaporin- 4 and the $\mathrm{K}+$ channel protein Kir4.1 differs in low- and high-grade human brain tumors. Acta Neuropathol (Berl) 2005; 109: 418-426

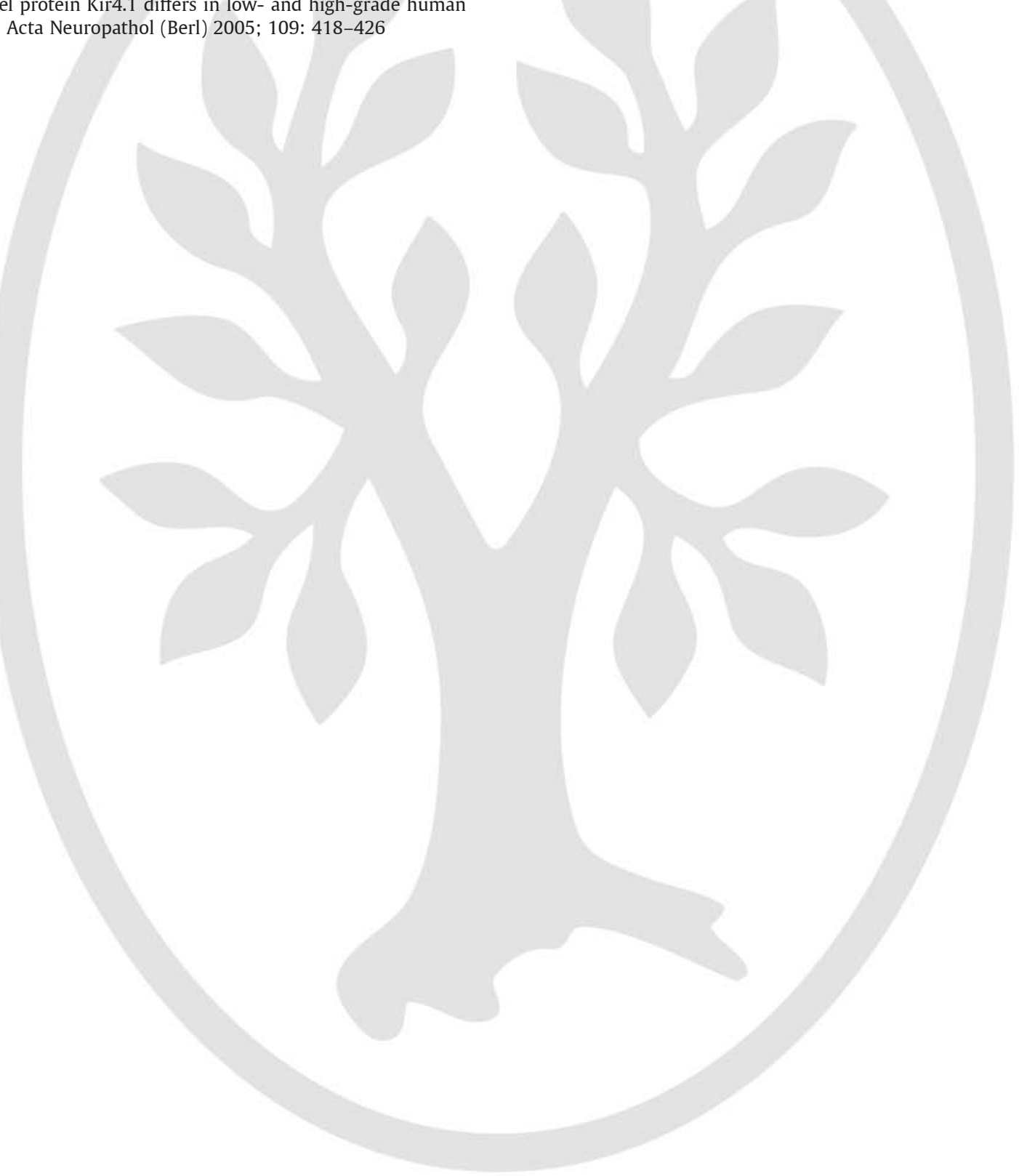

48 Watanabe A. Expression of syndecans, a heparan sulfate PG, in malignant gliomas: participation of nuclear factor-kappaB in upregulation of syndecan-1 expression. J Neurooncol 2006; 77: 25-33

49 Wiranowska M, Ladd S, Smith SR et al. CD44 adhesion molecule and neuro-glial PG NG2 as invasive markers of glioma. Brain Cell Biol 2006; 35: $159-172$

50 Xiang YY. Versican G3 domain regulates neurite growth and synaptic transmission of hippocampal neurons by activation of epidermal growth factor receptor. J Biol Chem 2006; 281: 19358-19368

51 Yee AJ, Akens M, Yang BL et al. The effect of versican G3 domain on local breast cancer invasiveness and bony metastasis. Breast Cancer Research 2007; 9: R47

52 Zagzag D, Friedlander DR, Dosik J et al. Tenascin-C expression by angiogenic vessels in human astrocytomas and by human brain endothelial cells in vitro. Cancer Res 1996; 56: 182-189

53 Zinzindohoué $F$, Lecomte Th, Ferraz J-M et al. Prognostic significance of MMP-1 and MMP-3 functional promoter polymorphisms in colorectal cancer. Clin Cancer Res 2005; 11: 594-599 